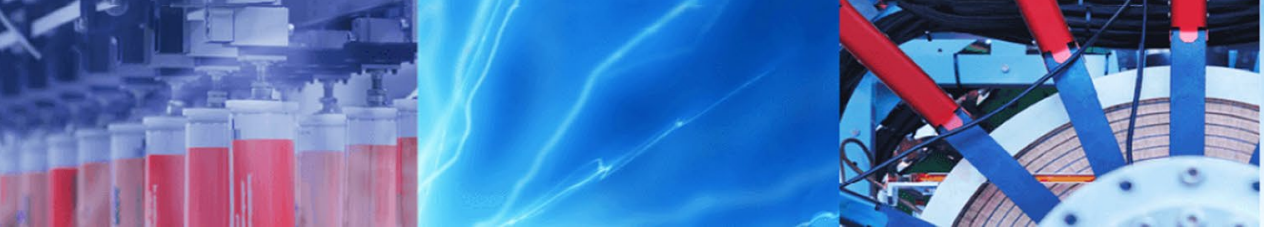

Research Article

\title{
Improved averaging techniques for solving multi-objective optimization (MOO) problems
}

\author{
Chandra Sen ${ }^{1}$
}

Received: 5 July 2019 / Accepted: 11 December 2019 / Published online: 29 January 2020

(c) Springer Nature Switzerland AG 2020

\begin{abstract}
This study evaluates the existing averaging techniques used for solving multi-objective optimization (MOO) problems. The problems are solved using mean, geometric and harmonic mean averaging techniques. The solutions obtained using the existing averaging techniques were not appropriate. Improved averaging techniques using mean, geometric and harmonic mean are proposed in this study. These techniques have been tested with the suitable examples and found superior to existing $\mathrm{MOO}$ averaging techniques.
\end{abstract}

Keywords Averaging technique $\cdot$ Improved averaging technique $\cdot$ Mean $\cdot$ Geometric mean $\cdot$ Harmonic mean

\section{Introduction}

Making an appropriate decision with conflicting objectives is not easy. Multi-objective optimization (MOO) techniques are helpful in improving the decision-making process in such a situation. Weighted sum techniques are more popular [1] in solving MOO problems. Several MOO averaging techniques [2-6] have been developed during the recent past. Most of these techniques have been tested with non conflicting objectives that were not appropriate. The MOO problem with multiple conflicting objectives is the prerequisite for the application of any MOO technique. Further the existing averaging techniques have been evaluated on the basis of the values of combined multi-objective function which was also not appropriate. The solutions should have been evaluated on the basis of the values of the original objective functions only. There are few more problems with these techniques $[1,7-9]$ in obtaining an acceptable solution of conflicting objectives. However the Sen's MOO technique [10] is efficient in generating appropriate solutions of the problems with conflicting objectives. This technique has been successfully applied for resource use planning in agriculture [11-21] with conflicting objectives of maximization of farm income and employment with minimum use of fertilizer, irrigation, plant protection chemicals, etc. However, the Sen's MOO technique have been declared inefficient in several studies using averaging techniques. After analyzing the comments and limitations of the prevailing averaging techniques, the improved average techniques are proposed in this study. Section 2 deals with the structure of $\mathrm{MOO}$ averaging techniques. The algorithms of averaging techniques have been presented in the Sect. 3. The examples solved with these techniques are given in Sect. 4. Section 5 deals with the discussion of the results and finally the findings are concluded in the last Sect. 6.

\section{MOO averaging techniques}

\subsection{Existing MOO averaging techniques}

For achieving ' $n$ ' objective functions with maximization of ' $r$ ' objective functions and minimization of remaining

Chandra Sen, chandra_sen@rediffmail.com | ${ }^{2}$ Department of Agricultural Economics, Institute of Agricultural Sciences, Banaras Hindu University, Varanasi 221005, India. 
' $n$-r' objective functions, the multi-objective optimization model is formulated as follows:

Optimize $Z=\left[\operatorname{Max} . Z_{1}, Z_{2} \ldots Z_{r}\right.$, Min. $\left.Z_{r+1}, \ldots Z_{n}\right]$

Subject to :

$A X=b$ and $X \geq 0$

\subsection{Individual optimization}

Individual optimization is a prerequisite for assessing the need for multi-objective optimization. First, all the ' $n$ ' objectives are optimized to obtain ' $n$ ' individual optimal solutions. There are ' $n$ ' optimal values of ' $n$ ' objective functions along with the ' $n$ ' set of values of decision variables $X_{i}$. Using each set of the values of decision variables, the suboptimal values of remaining $n-1$ objectives are estimated. The solution of individual optimization is arranged in an ' $n \times n$ ' matrix as shown in Table 1.

The columns are the optimal solutions of ' $n$ ' objective functions. The rows show the optimal and suboptimal values of all the objective functions. If all the columns contain the same values as in all the rows, the solutions are unique and multi-objective optimization is not required. When all ' $n$ ' or lesser solutions are different, multi-objective optimization is needed.

\subsection{Multi-objective function}

The multi-objective function is constructed by scalarizing each objective function by the mean, geometric and harmonic mean of optimal values of objective functions under the existing $\mathrm{MOO}$ averaging techniques as follows:
Maximize $\quad Z=\frac{\sum_{j=1}^{r} Z j}{\left|\theta_{1}\right|}-\frac{\sum_{j=r+1}^{s} Z j}{\left|\theta_{2}\right|}$

Subject to :

$$
\begin{aligned}
& A X=b \text { and } X \geq 0 \\
& \theta_{j} \neq 0 \text { for } J=1,2 \ldots s .
\end{aligned}
$$

where $\left|\theta_{1}\right|$, Mean, Geometric Mean and Harmonic Mean of optimal values of maximization objective functions. $\left|\theta_{2}\right|=$ Mean, Geometric Mean and Harmonic Mean of optimal values of Minimization Objective functions.

Note: The estimation of $\theta_{1}$ and $\theta_{2}$ is not logical when objective functions are of different dimensions.

Furthermore, the multi-objective function is formulated for the improved averaging techniques as detailed below:

Maximize $\quad \mathrm{Z}=\frac{\sum_{j=1}^{r} Z \mathrm{j}}{\left|\theta_{\mathrm{i}}\right|}-\frac{\sum_{j=r+1}^{s} Z \mathrm{j}}{\left|\theta_{\mathrm{j}}\right|}$

Subject to :

$$
\begin{aligned}
& A X=b \text { and } X \geq 0 \\
& \theta_{j} \neq 0 \text { for } J=1,2 \ldots s .
\end{aligned}
$$

where $\left|\theta_{i}\right|$, Mean, Geometric Mean and Harmonic Mean of optimal and suboptimal values of ith Maximization Objective function. $\left|\theta_{\mathrm{j}}\right|$, mean, Geometric Mean and Harmonic Mean of optimal and suboptimal values of jth Minimization Objective function.

\section{Solving MOO problems}

\subsection{Algorithm of existing averaging techniques}

An algorithm for solving $\mathrm{MOO}$ problems using averaging

\begin{tabular}{|c|c|c|c|c|c|c|c|}
\hline \multirow[t]{2}{*}{ Obj. function } & \multicolumn{7}{|c|}{ Individual optimization } \\
\hline & $\operatorname{Max} . Z_{1}$ & $\operatorname{Max} . Z_{2}$ & - & $\operatorname{Max} . Z_{r}$ & Min. $Z_{r+1}$ & - & $\operatorname{Min} . Z_{n}$ \\
\hline$Z_{1}$ & $\theta_{11}$ & $\theta_{12}$ & - & $\theta_{1 \mathrm{r}}$ & $\theta_{1 \mathrm{r}+1}$ & - & $\theta_{1 \mathrm{n}}$ \\
\hline$Z_{2}$ & $\theta_{21}$ & $\theta_{22}$ & - & $\theta_{21}$ & $\theta_{2 r+1}$ & - & $\theta_{2 n}$ \\
\hline- & - & - & - & - & - & - & - \\
\hline- & - & - & - & - & - & - & - \\
\hline $\mathrm{Z}_{\mathrm{r}}$ & $\theta_{\mathrm{r} 1}$ & $\theta_{\mathrm{r} 2}$ & - & $\theta_{\mathrm{rr}}$ & $\theta_{r r+1}$ & - & $\theta_{\mathrm{rn}}$ \\
\hline$Z_{r+1}$ & $\theta_{r+11}$ & $\theta_{r+12}$ & - & $\theta_{r+1 r}$ & $\theta_{r+1 r+1}$ & - & $\theta_{r+1 n}$ \\
\hline- & - & - & - & - & - & - & - \\
\hline$Z_{n}$ & $\theta_{\mathrm{n} 1}$ & $\theta_{\mathrm{n} 2}$ & - & $\theta_{\mathrm{nr}}$ & $\theta_{n r+1}$ & - & $\theta_{\mathrm{nn}}$ \\
\hline
\end{tabular}
techniques is given as:
Table 1 Individual optimization matrix $\theta_{\mathrm{ij}}=$ Value of ith objective function on the optimal solution of jth objective function

$\theta_{\mathrm{ii}}=$ Optimal value of the ith objective function 
Step I: Obtain optimal solution of all the objectives individually.

Step II: Calculate the averages of the optimal values of Maximization objective functions and Minimization objective function separately.

Step III: Divide all the maximization objective functions by the averages of individual optima of maximization objectives and minimization objective functions by the averages of the individual optima of minimization objectives.

Step IV: Sum the weighted maximization and minimization objective functions separately.

Step V: Construct the combined objective function by subtracting weighted minimization objective function from weighted maximization objective function.

Step VI: Maximize the combined objective function.

\subsection{Algorithm of improved averaging techniques}

The algorithm of improved averaging techniques is described below:

Step I: Same as 3.1

Step II: Calculate the averages of optimal and sub optimal values of each objective function separately.

Step III: Divide each objective function by the averages of its optimal and sub optimal values estimated in step II.

Step IV: Same as 3.1

Step V: Same as 3.1

Step VI: Same as 3.1

\section{Numerical examples}

Example 1 The example of the study [6] has been reproduced here for present analysis.

$$
\begin{aligned}
& \text { Max. } Z_{1}=12500 X_{1}+25100 X_{2}+16700 X_{3}+23300 X_{4}+20200 X_{5} \\
& \text { Max. } Z_{2}=21 X_{1}+15 X_{2}+13 X_{3}+17 X_{4}+11 X_{5} \\
& \text { Min. } Z_{3}=370 X_{1}+280 X_{2}+350 X_{3}+270 X_{4}+240 X_{5} \\
& \text { Min. } Z_{4}=1930 X_{1}+1790 X_{2}+1520 X_{3}+1690 X_{4}+1720 X_{5} \\
& \text { Subject to : } \\
& X_{1}+X_{2}+X_{3}+X_{4}+X_{5}=4.5 \\
& \qquad \begin{array}{c}
2 X_{1} \geq 1.0 \\
\quad 3 X_{4} \geq 1.5
\end{array}
\end{aligned}
$$

\subsection{Solution}

The example was solved for achieving each objective and the results are presented in Table 2 . Each column gives the optimal value of the mentioned objective function and suboptimal values of the remaining objective functions. The values of decision variables are given in the $X_{i}$ row.
Table 2 Individual optimization matrix

\begin{tabular}{lllll}
\hline Item & \multicolumn{4}{l}{ Individual optimization } \\
\cline { 2 - 5 } & Max. $Z_{1}$ & Max. $Z_{2}$ & Min. $Z_{3}$ & Min. $Z_{4}$ \\
\hline $\mathrm{Xi}$ & $\begin{array}{c}0.5,3.5,0, \\
0.5,0\end{array}$ & $4,0,0,0.5,0$ & $0.5,0,0,0.5$, & $0.5,0,3.5,0.5,0$ \\
& \multicolumn{4}{l}{3.5} \\
$Z_{1}$ & 105750 & 61650 & 88600 & 76350 \\
$Z_{2}$ & 71.5 & 92.5 & 57.5 & 64.5 \\
$Z_{3}$ & 1300 & 1615 & 1160 & 1545 \\
$Z_{4}$ & 8075 & 8565 & 7830 & 7130 \\
\hline
\end{tabular}

The maximum value of $Z_{1}$ is 105750 obtained on $X_{1}=0.5$, $X_{2}=3.5$ and $X_{4}=0.5$. On the same solution the remaining objective functions $Z_{2}, Z_{3}$ and $Z_{4}$ got suboptimal values of $Z_{2}=71.5, Z_{3}=1300$ and $Z_{4}=8075$. Similar results have been observed in optimization of objective function $Z_{2}$, $Z_{3}$ and $Z_{4}$. All the solutions of individual optimizations are different. None of the solutions optimize all the objective functions simultaneously. The conflicts among objective functions clearly necessitate the use of multi-objective optimization.

The existing $\mathrm{MOO}$ averaging techniques using mean, geometric mean and harmonic mean have been applied for solving the Example 1. The example was also solved using the improved averaging techniques with mean, geometric mean and harmonic mean for comparison. The estimation of mean, geometric mean and harmonic mean for both existing and improved techniques are shown in Table 3. Under the improved averaging techniques, all the optimal and suboptimal values of each objective function in a row have been considered for estimation of mean, geometric and harmonic mean. For the existing averaging techniques, only optimal values of the objective functions of maximization and minimization have been considered separately for the estimation of mean, geometric and harmonic mean.

The example was solved with the existing as well as improved averaging techniques using mean, geometric and harmonic mean for formulation of multi-objective functions. The solution of multi-objective optimization is presented in Table 4.

The results of multi-objective optimization with existing averaging techniques using mean, geometric and harmonic mean are all the same and achieve the first objective only. The remaining three objectives are not met. However, the improved averaging techniques have generated a compromising solution in achieving all the four objectives simultaneously. The improved averaging techniques have generated a comprehensive more acceptable solution than the existing averaging techniques. All these averaging techniques have been tested again on another example as described below. 
Example 2 The example has been taken from the study [5] of multi-objective programming.

Max. $Z_{1}=7000 X_{1}+10000 X_{2}+4000 X_{3}+5000 X_{4}+9000 X_{5}$

Max. $Z_{2}=89 X_{1}+50 X_{2}+100 X_{3}+79 X_{4}+95 X_{5}$

Min. $Z_{3}=10 X_{1}+15 X_{2}+14 X_{3}+13 X_{4}+12 X_{5}$

Min. $Z_{4}=150 X_{1}+120 X_{2}+140 X_{3}+100 X_{4}+130 X_{5}$

Subject to :

$x_{1}+x_{2}+x_{3}+x_{4}+x_{5}=7$

$\mathrm{X}_{1} \geq 0.7$

$x_{2} \geq 0.3$

$x_{3} \geq 0.4$

$X_{5} \geq 0.6$

\subsection{Solution}

All the objectives have been optimized individually to find the optimal values of each objective. The results are presented in Table 5. Each optimal solution achieved the single objective only ignoring the remaining three objectives. The solution indicates the presence of conflicts among all the four objectives. The mean, geometric mean and harmonic mean of optimal values are estimated and given in Table 6.

The multi-objective functions for both existing and improved averaging techniques have been formulated and optimized with given constraints. The results of both the techniques are presented in the Table 7. The existing averaging techniques are achieving the first objective only. Whereas, all the four objectives have been achieved simultaneously by the improved averaging techniques.

\section{Discussion}

It is clear from the results of both the examples that the improved averaging techniques are efficient in solving MOO problems. The solutions of multi objective optimization of both the examples 1 and 2 indicate that all the existing averaging techniques are unable to provide the compromising solutions. These solutions have achieved the first objective only ignoring all the remaining three objectives. However, the improved averaging techniques have achieved all the four objectives simultaneously.
Table 3 Mean, geometric mean and harmonic mean

\begin{tabular}{|c|c|c|c|c|c|c|}
\hline \multirow{2}{*}{$\begin{array}{l}\text { Objective } \\
\text { function }\end{array}$} & \multicolumn{3}{|c|}{ Existing averaging techniques } & \multicolumn{3}{|c|}{ Improved averaging techniques } \\
\hline & Mean & G. Mean & H. Mean & Mean & G. Mean & H. Mean \\
\hline $\mathrm{Z}_{1}$ & 52921.25 & 3127.59 & 184.83 & 83087.5 & 81491.82 & 79902.32 \\
\hline $\mathrm{Z}_{2}$ & & & & 71.5 & 70.37 & 69.33 \\
\hline$Z_{3}$ & 4145 & 2875.89 & 1995.36 & 1405 & 1392.75 & 1380.38 \\
\hline $\mathrm{Z}_{4}$ & & & & 7900 & 7882.79 & 7865.35 \\
\hline
\end{tabular}

Table 4 Multi-objective optimization

\begin{tabular}{|c|c|c|c|c|c|c|}
\hline \multirow[t]{2}{*}{ Item } & \multicolumn{3}{|c|}{ Existing averaging techniques } & \multicolumn{3}{|c|}{ Improved averaging techniques } \\
\hline & Mean & Harmonic mean & Geometric mean & Mean & Harmonic mean & Geometric mean \\
\hline Xi & $0.5,3.5,0,0.5,0$ & $0.5,3.5,0,0.5,0$ & $0.5,3.5,0,0.5,0$ & $0.5,0,0,4,0$ & $0.5,0,0,4,0$ & $0.5,0,0,4,0$ \\
\hline $\mathrm{Z}_{1}$ & 105750 & 105750 & 105750 & 99450 & 99450 & 99450 \\
\hline $\mathrm{Z}_{2}$ & 71.5 & 71.5 & 71.5 & 78.5 & 78.5 & 78.5 \\
\hline $\mathrm{Z}_{3}$ & 1300 & 1300 & 1300 & 1265 & 1265 & 1265 \\
\hline $\mathrm{Z}_{4}$ & 8075 & 8075 & 8075 & 7725 & 7725 & 7725 \\
\hline
\end{tabular}

Table 5 Individual optimization

\begin{tabular}{lllll}
\hline Item & \multicolumn{3}{l}{ Individual optimization } & \\
\cline { 2 - 5 } & Max. $Z_{1}$ & Max. $Z_{2}$ & Min. $Z_{3}$ & Min. $Z_{4}$ \\
\hline$X_{i}$ & $0.7,5.3,0.4,0,0.6$ & $0.7,0.3,5.4,0,0.6$ & $5.7,0.3,0.4,0,0.6$ & $0.7,0.3,0.4,5.0,0.6$ \\
$Z_{1}$ & 64900 & 34900 & 49900 & 39900 \\
$Z_{2}$ & 424.3 & 674.3 & 619.3 & 569.3 \\
$Z_{3}$ & 99.3 & 94.3 & 74.3 & 89.3 \\
$Z_{4}$ & 875 & 975 & 1025 & 775 \\
\hline
\end{tabular}


Table 6 Mean, geometric mean and harmonic mean

\begin{tabular}{|c|c|c|c|c|c|c|}
\hline \multirow{2}{*}{$\begin{array}{l}\text { Objective } \\
\text { function }\end{array}$} & \multicolumn{3}{|c|}{ Existing averaging techniques } & \multicolumn{3}{|c|}{ Improved averaging techniques } \\
\hline & Mean & G. Mean & H. Mean & Mean & G. Mean & H. Mean \\
\hline$Z_{1}$ & 32787.15 & 6615.29 & 1334.73 & 47400 & 46082.48 & 44860.98 \\
\hline $\mathrm{Z}_{2}$ & & & & 571.8 & 563.56 & 554.70 \\
\hline$Z_{3}$ & 424.65 & 239.96 & 135.59 & 89.3 & 88.78 & 88.24 \\
\hline $\mathrm{Z}_{4}$ & & & & 912.5 & 907.32 & 902.03 \\
\hline
\end{tabular}

Table 7 Multi-objective optimization

\begin{tabular}{|c|c|c|c|c|c|c|}
\hline \multirow[t]{2}{*}{ Item } & \multicolumn{3}{|c|}{ Existing averaging techniques } & \multicolumn{3}{|c|}{ Improved averaging techniques } \\
\hline & Mean & Harmonic mean & Geometric mean & Mean & Harmonic mean & Geometric mean \\
\hline $\mathrm{Xi}$ & $0.7,5.3,0.4,0,0.6$ & $0.7,5.3,0.4,0,0.6$ & $0.7,5.3,0.4,0,0.6$ & $0.7,0.3,0.4,0,5.6$ & $0.7,0.3,0.4,0,5.6$ & $0.7,0.3,0.4,0,5.6$ \\
\hline $\mathrm{Z}_{1}$ & 64900 & 64900 & 64900 & 59900 & 59900 & 59900 \\
\hline$Z_{2}$ & 424.3 & 424.3 & 424.3 & 649 & 649 & 649 \\
\hline$Z_{3}$ & 99.3 & 99.3 & 99.3 & 84.3 & 84.3 & 84.3 \\
\hline $\mathrm{Z}_{4}$ & 875 & 875 & 875 & 925 & 925 & 925 \\
\hline
\end{tabular}

\section{Conclusion}

Existing and improved averaging MOO techniques using mean, geometric and harmonic mean have been applied for solving two examples of multi-objective optimization problem. The results show the superiority of the improved averaging techniques over existing averaging techniques for solving multi-objective optimization problems.

\section{Compliance with ethical standards}

Conflict of interest The author declares that there is no conflict of interest.

\section{References}

1. Timothy MR, Singh AJ (2004) Survey of multi-objective ptimization methods for engineering. Struct Multidiscip Optim 26(6):369-395

2. Sulaiman NA, Abdulrahim BK (2013) Using transformation technique to solve multi-objective linear fractional programming problem. Int J Res Rev Appl Sci 14(3):559-567

3. Huma A, Geeta M, Sushma D (2017) Transforming and optimizing multi-objective quadratic fractional programming problem. Int J Stat Appl Math 2(1):01-05

4. Nahar S, Abdul Alim Md (2017) A new statistical averaging method to solve multi-objective linear programming problem. Int J Sci Res 6(8):623-629

5. Othman A, Ameen H (2017) An adaptive arithmetic average transformation technique. LAMBERT Academic Publishing, Saarbrücken

6. Sohag Zl, Asadujjaman Md (2018) A proposed new average method for solving multi-objective linear programming problem using various kinds of mean techniques. Math Lett $4(2): 25-33$

7. Sen C (2018) Sen's multi-objective programming method and its comparison with other techniques. Am J Oper Res $8(1): 10-13$

8. Sen C (2018) Multi-objective optimization techniques: misconceptions and clarifications. Int J Sci Innov Math Res 6(6):29-33

9. Sen C (2019) Improved scalarizing techniques for solving multiobjective optimization problems. Am J Oper Res 9(1):8-11

10. Sen C (1983) A new approach for multi-objective rural development planning. Indian Econ J 30(4):91-96

11. Gangwar LS (1994) Technological advancements and its implications on sustainable agriculture-a case study of Nainital Tarai of Uttar Pradesh. Ph.D. thesis, Department of Agricultural Economics, Institute of Agricultural Sciences, Banaras Hindu University, Varanasi, India

12. Kushwaha S, Ochi JE (1996) Economic simulation to alternative resource use for sustainable agriculture in Nigeria-Sen's MOP approach. J Sci Res 46(1):105-114

13. Gautam K (2013) Natural and human resource use planning for Vindhyan Region of Eastern Uttar Pradesh. Ph.D. thesis, Department of Agricultural Economics, Institute of Agricultural Sciences, Banaras Hindu University, Varanasi, India

14. Kumar $\mathrm{H}$ (2012) Economic analysis of fresh water aquaculture in Maharajganj district of Eastern Uttar Pradesh. Ph.D. thesis, Department of Agricultural Economics, Institute of Agricultural Sciences, Banaras Hindu University, Varanasi, India

15. Kumari M, Singh OP, Meena DC (2017) Optimizing cropping pattern in Eastern Uttar Pradesh using Sen's multi objective programming approach. Agric Econ Res Rev 30(2):285-295

16. Sen C, Dubey PP (1994) Resource use planning in agriculture with single and multi-objective programming approaches (a comparative study). J Sci Res 44:75-81

17. Singh PK (2005) Multilevel multi-objective planning for Agriculture for district Mau, U.P. Ph.D. thesis. Department of Agricultural Economics, Institute of Agricultural Sciences, Banaras Hindu University, Varanasi, India

18. Singh VK (2002) Agricultural development and environmental pollution-a case study of District Varanasi. PhD thesis, Department of Agricultural Economics, Institute of Agricultural Sciences, Banaras Hindu University, Varanasi, India 
19. Sinha HK (2002) Effect of air pollution on peri-urban agriculture in Varanasi. PhD thesis, Department of Agricultural Economics, Institute of Agricultural Sciences, Banaras Hindu University, Varanasi, India

20. Gwandi O, Kamalvanshi V, Maurya MK, Kushuwaha S (2019) Farmers' livelihood strategies by optimizing resource use in farming of District Varanasi, Uttar Pradesh: application of Sen's multi-objective programming approach. Res J Agric Sci 10(1):116-119
21. Kumar H, Singh R, Verma SC, Pal VK (2019) Multi-objective programming (MOP) model for aquaculture production systems in the Northern India. Int J Res Eng Sci Manag 2(4):295-298

Publisher's Note Springer Nature remains neutral with regard to jurisdictional claims in published maps and institutional affiliations. 